\title{
Magnetic nanoparticles in cancer therapy: how can thermal approaches help?
}

\author{
"The synergistic effect of magnetic hyperthermia and \\ photothermia has been previously reported for nanohybrids. \\ Yet, while imagination seems the limit to the choice of \\ materials (and material combinations) and architectures of \\ nanoparticles ... clinical requirements remain very strict... It \\ is therefore essential to focus on reasonable options, which \\ already had involved and probably will still include iron oxide \\ nanoparticles."
}

First draft submitted: 9 January 2017; Accepted for publication: 12 January 2017; Published online: 21 February 2017

Keywords: cancer $\bullet$ combined nanotherapy $\bullet$ iron oxide nanoparticles $\bullet$ magnetic hyperthermia • photothermal therapy

The limitations of conventional anticancer therapies (such as moderate therapeutic efficacy, drug resistance, severe adverse reactions, low concentration of therapeutics at the site of action and/or conventional drugs fast renal clearance, etc.) [1] call for novel and often combined anticancer approaches. chemotherapy and radiation therapy are thus increasingly combined with hyperthermia, a local, regional or whole body heating, obtained by microwave, radiofrequency, ultrasound energy or, more recently, magnetism.

Magnetic hyperthermia relies on the concept that large doses (as sufficient heat could only be obtained by massive nanoparticles administrations) of intratumorally injected magnetic nanoparticles may generate heat after exposure to an external alternating magnetic field and consequently induce cell death within unresectable tumors $[2,3]$. This method has been used thus far in clinics [4] to treat terminal illness (mainly prostate tumors and glioblastoma multiforme) and the major advantages of this technique are its minimal invasiveness (as the approach requires a single injection of nanoparticles suspension) and its remote application to deep-seated tumors.

\section{Versatility of iron oxide nanoparticles}

Amid prospective therapeutics for magnetic hyperthermia, inorganic magnetic-nanoparticle-based nanomaterials, especially the iron oxide ones, appear particularly appealing because they might be applied in multifunctional approaches (i.e., therapy and diagnosis) and could be used for multimodal therapeutic strategies (e.g., magnetic and photothermal). Besides, the particles could benefit from a large variety of distinct technical improvements, as they offer relatively large platforms to which different materials or chemical moieties could be attached. This might increase particles circulating half-lives, allow specific targeting and/or modify activation onsets triggered by external stimuli. A one and only agent could thereafter be used and applied in one single dose, to destroy cancer cells and disorganize cancer cells environment by physical and chemical means. In addition, the treatment could be ignited either by consecutive onsets or set on by simultaneous heating sources in order to optimize the synergistic efficiency of multiple (thermal) effects.

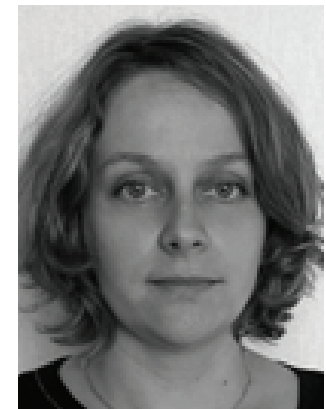

Jelena Kolosnjaj-Tabi Author for correspondence: Laboratoire Matière et Systèmes Complexes MSC, UMR 7057, CNRS \& University Paris Diderot, 75205 Paris Cedex 13, France

kolosnjaj@gmail.com

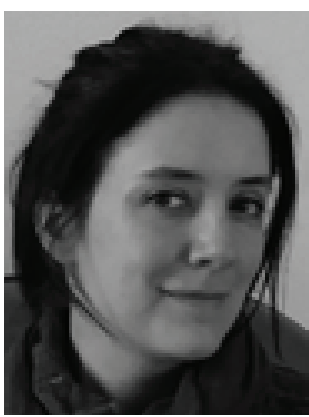

Claire Wilhelm

Author for correspondence:

Laboratoire Matière et Systèmes Complexes MSC, UMR 7057, CNRS \& University Paris Diderot, 75205 Paris Cedex 13, France

claire.wilhelm@univ-paris-diderot.fr

Future $\because \%$ Medicine part of 


\section{Multiple targets of (magneto)thermal therapy}

Hyperthermia-induced cell death was often linked to the apoptosis of cancer cells $[5,6]$ lying near substantial particles injections, but could be linked also to an immunogenic response to cells lying away from the particles, as dying cells triggered an immune response [7]. Moreover, additional mechanisms of action have recently been suggested, as magnetic nanoparticles can also generate heat at the intracellular level (within the lysosomes) [8]. This can lead to cell death involving lysosomal death pathways, suggesting that magnetic hyperthermia could also be applicable to apoptosisresistant cancer cells [8]. Finally, heating nanoparticles also affect the tumoral stroma [9]. The latter, rich in collagen fibers, might be disorganized by the heat (as collagen fibers slacken due to their heat-dependent phase transition). This results in increased penetration of nanoparticles themselves and also increases the permeation of drugs (e.g., doxorubicin) throughout the tumor [9].

\section{Improving the heating performance}

In order to optimize the therapy by increasing the heating potential of magnetic nanoparticles (thus allow decreasing their dosage), new trends in nanodesign have emerged.

They consist in optimizing nanoparticles synthesis, as tuning their size and anisotropy improves the heating properties of iron oxide crystals [10-12]. In addition, particles can be complemented, generally with (multiple) inorganic cores/shells made of other constituents. The latter often include plasmonic materials, in other words, gold or silver $[13,14]$ or semiconducting carbon nanotubes and graphene [15,16]. The so obtained nanohybrids could be activated by other (additional) sources, such as light, and thus extend nanoparticles use to photothermia. But is this composite ado really necessary?

We have recently reported on the amplification of the heating potential of iron oxide nanoparticles by combining the alternating magnetic field and the near-infrared light [17]. These two physical approaches implicating two excitation sources led to a heating capacity of up to $5000 \mathrm{~W} / \mathrm{g}$, which was obtained by a single heat-generating material, the (mono composite) cubic iron oxide nanocrystals [17]. To provide a specific comparison, the heat obtained by this bi-modal heating is 100 -fold greater than what is generally applied in regional radiofrequency hyperthermia.

This extremely high heating potential was achieved within nanoparticles aqueous suspensions and in vitro within cancer cells. While single mode treatment of either magnetic hyperthermia or photothermia reduced tumor growth to a moderate extent, the heat applied in the combined treatment resulted in complete tumor regression in all treated animals [17]. The nanoparticles that were used for this pilot study had an optimized crystal structure and were only coated with a PEG-gallol coating. We do, however, anticipate that further surface modification and functionalization could, eventually, broaden such nanoparticles used to more challenging cancer types and allow applications of particles via other routes of administration (i.e., intravenous or nasal).

\section{The importance of 'keeping it simple'}

The synergistic effect of magnetic hyperthermia and photothermia has been previously reported for nanohybrids. Yet, while imagination seems the limit to the choice of materials (and material combinations) and architectures of nanoparticles, resulting in fancy designs and fantastic chemical and physical properties, clinical requirements remain very strict and the bench to bedside transition implies long, rigorous and complex regulatory processes. It is therefore essential to focus on reasonable options, which already had involved and probably will still include iron oxide nanoparticles [18]. The latter not only have a wellestablished fate and good biocompatibility and biodegradability $[19,20]$, but have also been shown to act as extremely effective magnetic and photo-responsive sources of heat $[17]$.

\section{Future perspective}

Despite aborted attempts in the marketing of magnetic iron oxide nanoparticles, because they generated low profit as contrast agents for magnetic resonance imaging, latest findings indicate that iron oxide nanoparticles still did not have the last word. Current approaches now accentuate their use for nanoparticle-mediated magnetic hyperthermia combined with photothermal therapy, for which they seem to offer an unrivalled potential in terms of intratissular heat delivery. Finally, these multifunctional nanoparticles could also be further customized to offer specific targeting, that could, with some scientific effort, reach more complex targets and, at some point, cure metastases after a completely noninvasive systemic administration.

\section{Financial \& competing interests disclosure}

The authors have no relevant affiliations or financial involvement with any organization or entity with a financial interest in or financial conflict with the subject matter or materials discussed in the manuscript. This includes employment, consultancies, honoraria, stock ownership or options, expert testimony, grants or patents received or pending, or royalties.

No writing assistance was utilized in the production of this manuscript. 


\section{References}

1 Lammers T, Kiessling F, Hennink WE, Storm G. Drug targeting to tumors: principles, pitfalls and (pre-) clinical progress. J. Control. Release 161(2), 175-187 (2012).

2 Hilger I. In vivo applications of magnetic nanoparticle hyperthermia. Int. J. Hyperthermia 29(8), 828-834 (2013).

3 Hilger I, Kaiser WA. Iron oxide-based nanostructures for MRI and magnetic hyperthermia. Nanomedicine 7(9), 1443-1459 (2012).

4 Thiesen B, Jordan A. Clinical applications of magnetic nanoparticles for hyperthermia. Int. J. Hyperthermia 24(6), 467-474 (2008).

5 Moros M, Ambrosone A, Stepien G et al. Deciphering intracellular events triggered by mild magnetic hyperthermia in vitro and in vivo. Nanomedicine 10(14), 2167-2183 (2015).

6 Blanco-Andujar C, Ortega D, Southern P, Nesbitt SA, Thanh NTK, Pankhurst QA. Real-time tracking of delayed-onset cellular apoptosis induced by intracellular magnetic hyperthermia. Nanomedicine 11(2), 121-136 (2016).

7 Kobayashi T, Kakimi K, Nakayama E, Jimbow K. Antitumor immunity by magnetic nanoparticle-mediated hyperthermia. Nanomedicine 9(11), 1715-1726 (2014).

8 Sanchez C, El Hajj Diab D, Connord V et al. Targeting a $\mathrm{G}$-protein-coupled receptor overexpressed in endocrine tumors by magnetic nanoparticles to induce cell death. ACS Nano 8(2), 1350-1363 (2014).

9 Kolosnjaj-Tabi J, Di Corato R, Lartigue L et al. Heatgenerating iron oxide nanocubes: subtle "destructurators" of the tumoral microenvironment. ACS Nano 8(5), 4268-4283 (2014).

10 Gazeau F, Lévy M, Wilhelm C. Optimizing magnetic nanoparticle design for nanothermotherapy. Nanomedicine (Lond.) 3(6), 831-844 (2008).
11 Périgo EA, Hemery G, Sandre O et al. Fundamentals and advances in magnetic hyperthermia. Appl. Phys. Rev. 2(4), 041302 (2015).

12 Blanco-Andujar C, Walter A, Cotin G et al. Design of iron oxide-based nanoparticles for MRI and magnetic hyperthermia. Nanomedicine 11(14), 1889-1910 (2016).

13 Espinosa A, Bugnet M, Radtke G et al. Can magnetoplasmonic nanohybrids efficiently combine photothermia with magnetic hyperthermia? Nanoscale 7(45), 18872-18877 (2015).

$14 \mathrm{Xu}$ Z, Hou Y, Sun S. Magnetic core/shell Fe3O4/Au and $\mathrm{Fe} 3 \mathrm{O} 4 / \mathrm{Au} / \mathrm{Ag}$ nanoparticles with tunable plasmonic properties. J. Am. Chem. Soc. 129(28), 8698-8699 (2007).

15 Ma X, Tao H, Yang K et al. A functionalized graphene oxide-iron oxide nanocomposite for magnetically targeted drug delivery, photothermal therapy, and magnetic resonance imaging. Nano Res. 5(3), 199-212 (2012).

16 Yang K, Hu L, Ma X et al. Multimodal imaging guided photothermal therapy using functionalized graphene nanosheets anchored with magnetic nanoparticles. $A d v$. Mater. 24(14), 1868-1872 (2012).

17 Espinosa A, Di Corato R, Kolosnjaj-Tabi J, Flaud P, Pellegrino T, Wilhelm C. Duality of iron oxide nanoparticles in cancer therapy: amplification of heating efficiency by magnetic hyperthermia and photothermal bimodal treatment. ACS Nano 10(2), 2436-2446 (2016).

18 Singh D, Mcmillan JM, Kabanov AV, Sokolsky-Papkov M, Gendelman HE. Bench-to-bedside translation of magnetic nanoparticles. Nanomedicine 9(4), 501-516 (2014).

19 Ling D, Hyeon T. Chemical design of biocompatible iron oxide nanoparticles for medical applications. Small 9(9-10), 1450-1466 (2013).

20 Mazuel F, Espinosa A, Luciani N et al. Massive intracellular biodegradation of iron oxide nanoparticles evidenced magnetically at single-endosome and tissue levels. ACS Nano 10(8), 7627-7638 (2016). 